\title{
Evaluation of Inbred Lines of Baby Corn Through Line $x$ Tester Method
}

\author{
Shahnewaz Begum, Mohammad Amiruzzaman, Mohammad Quamrul Islam Matin, \\ Sumaiya Haque Omy, Md. Motiar Rohman
}

Plant Breeding Division, Bangladesh Agricultural Research Institute, Gazipur, Bangladesh

\section{Email address:}

shahnewaz_ctg1952@yahoo.com (S. Begum), amiruzzaman95@yahoo.com (M. Amiruzzaman), quamrul_islam76@yahoo.com (M. Q. I. Matin), saumi77@gmail.com (S. H. Omy), motiar_1@yahoo.com (M. M. Rohman)

\section{To cite this article:}

Shahnewaz Begum, Mohammad Amiruzzaman, Mohammad Quamrul Islam Matin, Sumaiya Haque Omy, Md. Motiar Rohman. Evaluation of Inbred Lines of Baby Corn Through Line $\times$ Tester Method. Plant. Vol. 4, No. 6, 2016, pp. 101-107. doi: 10.11648/j.plant.20160406.17

Received: September 26, 2016; Accepted: October 10, 2016; Published: November 3, 2016

\begin{abstract}
Eight $\mathrm{S}_{3}$ baby corn lines of KH101 were evaluated following line $(8) \times$ tester $(3)$ method by determining general combining ability (GCA) and specific combining ability (SCA). Highly significant genotypic differences were observed indicated wide range of variability present among the genotypes. Variance due to SCA was larger than GCA variance for all the characters indicating the preponderance of non-additive gene action in the expression of various traits. Among the parents none were found as good general combiners for baby corn yield. None of 24 cross combinations showed significant SCA effects for yield per plant. Considering cob length, cob diameter, cob per plant, total fodder weight and yield per plant the crosses $\mathrm{KH}-$ $101 / \mathrm{S}_{3}-44 \times \mathrm{VS} / \mathrm{S}_{3}-24$ and $\mathrm{KH}-101 / \mathrm{S}_{3}-44 \times \mathrm{VS} / \mathrm{S}_{3}-25$ were selected as promising baby corn hybrids.
\end{abstract}

Keywords: Evaluation, Inbred, Baby Corn, Line $\times$ Tester, SCA, GCA

\section{Introduction}

Baby corn are young and unfertilized corn ears with 2-3 $\mathrm{cm}$ emerged silks. Baby corn can be eaten raw and included in the diet in manifold ways, e.g., in salad, chutney, pakora, soup, preserves and so on. Baby corn, popular in many Asian cuisines, has become a staple in salad bars across the United States. Most of the baby corn sold in the US and in Europe is imported from Thailand, Taiwan and Indonesia. It is an extremely easy crop to produce and is grown just like any other corn crop. It is not produced locally because hand labor is required for harvesting and processing, market prices are unknown, and consumers are unfamiliar with it as a fresh crop. However, locally produced fresh baby corn has several advantages over imported baby corn. It is superior in both taste and texture to processed baby corn and it can easily be grown organically. There has been a large increase in demand for organic foods. This may be the time for small farmers to test the market for baby corn locally. Markets may include organic sales through farmers' markets, restaurants, local grocery stores and health food stores ([1]. Despite manifold uses of baby corn, very little information on breeding strategies followed for improvement in baby corn [2]. Due to the limited number of studies on baby corn, no high-yielding cultivars were developed for the market. Currently some early-maturing corn cultivars, originally destined for grain production, are used for baby corn production [3]. Breeders obtain abundant information from line $\times$ tester analysis by Kempthorne [4], which is often used in breeding programs of different crops, due to its efficiency in selecting parents for crosses resulting in hybrids with desirable phenotypes. This analysis provides estimates of general (GCA) and specific (SCA) combining ability as well as information about the predominance of genes with additive and non-additive effects in the trait control [5]. The aim of this study was to evaluate the combining ability of baby corn lines and hybrids with favorable traits for the production of baby corn in lines derived from commercial hybrid.

\section{Materials and Methods}

Seeds were sown following Randomized Complete Block Design (RCBD) with two replications at Bangladesh Agricultural Research Institute, Joydebpur during rabi, 201415. Spacing was maintained at $60 \mathrm{~cm} \times 20 \mathrm{~cm}$. Two border 
rows were used at each end of the replication to minimize the border effect. Fertilizer were applied @ 250, 55, 110, 40, 5 and $1.5 \mathrm{Kg} /$ ha of $\mathrm{N}, \mathrm{P}, \mathrm{K}, \mathrm{S}, \mathrm{Zn}$ and $\mathrm{B}$, respectively. All the recommended packages of practice were followed and the observations were recorded on ten randomly selected plants for quantitative characters viz. days to $50 \%$ tasselling, days to $50 \%$ silking, plant height $(\mathrm{cm})$, upper cob height $(\mathrm{cm})$, lower cob height $(\mathrm{cm})$, weight of per cob with husk $(\mathrm{g})$, weight of per cob without husk $(\mathrm{g})$, cob length $(\mathrm{cm})$, cob diameter $(\mathrm{cm})$, number of cob per plant, fodder yield per plant $(\mathrm{g})$, days to 1 st cob harvest, interval between 1 st and last cob harvest and cob yield per plant (g). Estimates of combining ability and their variance were made as suggested by Kempthorne [4].

\section{Results and Discussions}

\subsection{Analysis of Variance}

The analysis of variance revealed significant differences among the crosses for yield and other traits indicating sufficient genetic variability present among the hybrids. The analysis of variance also revealed significant differences in the variance due to lines, testers, and line $\times$ tester for yield and some yield contributing traits (Table 1). Significant differences were observed among the lines for weight of per cob with husk, cob length $(\mathrm{cm})$, cob diameter $(\mathrm{cm})$, number of cob per plant, days to 1 st cob harvest and yield per plant (g). Noteworthy difference was also exist among testers for weight of per cob with husk, weight of per cob without husk, days to $50 \%$ silking, number of cob per plant, total fodder weight per plant $(\mathrm{g})$ and cob yield per plant $(\mathrm{g})$. Significant variances were also observed in interactions of line $\times$ testers for weight of per cob with husk, weight of per cob without husk, number of cob per plant, total fodder weight per plant (g) and cob yield per plant $(\mathrm{g})$ indicating that there were wide range of variability among lines, testers and their interactions for the traits under study.

Higher estimation of dominance variance as compared to additive variance for all the characters indicates the predominant role of non-additive type of gene action play in the inheritance which suggests the scope of improvement of these characters through heterosis breeding. Similar finding were reported by Ceyhan et al. [6], Kanagarasu et al. [7] and Motamedi et al. [8] and Ahmed et al. [9] in corn for different characters. Involvement of non-additive gene action for the characters in present investigation is also in consonance with the findings of Suneetha et al. [10] for days to $50 \%$ tasselling and silking, Dhasarathan et al. [11] for days to 50\% tasseling, plant height, number of baby corns per plant, baby corn length, baby corn weight, Anantha [12] and Selvarani [13] for days to tasseling, Geetha and Jayaraman [14], Anantha [12] and Prakash and Ganguli [15] for plant height, Rodrigues and Da Silva [16] for baby corn length, Mahto and Ganguly [17] for grain yield.

Table 1. Mean squares and estimation of variance for different characters of baby corn.

\begin{tabular}{|c|c|c|c|c|c|c|c|c|}
\hline Source & df & DT & DS & PH (cm) & $\mathrm{UCH}(\mathrm{cm})$ & LCH (cm) & WPCH (g) & WPCWH (g) \\
\hline Replications & 1 & 91.4 & $98.4 * *$ & 806 & 88.4 & 283 & 25.8 & 8.24 \\
\hline Treatments & 34 & $38.6^{* *}$ & $45.0 * *$ & $1672 * *$ & $943 * *$ & $559 * *$ & 218 & 8.69 \\
\hline Parents & 10 & 23.2 & $12.3 * *$ & $501 *$ & $311 * *$ & $97.5 * *$ & $443 * *$ & $20.6 * *$ \\
\hline Parents vs Crosses & 1 & $891 * *$ & $1105^{* *}$ & $4657 * *$ & $21245^{* *}$ & 11955 & $130 * *$ & $20.7 * *$ \\
\hline Crosses & 23 & 8.26 & 13.1 & 228 & 335 & $264 * *$ & $123 * *$ & 2.99 \\
\hline Lines & 7 & 6.94 & 10.5 & 132 & $61.2 *$ & 132 & $230 * *$ & 3.27 \\
\hline Testers & 2 & 26.4 & 65.0 & 1416 & $3315^{*}$ & $1753 * *$ & $97.3 * *$ & $0.67 * *$ \\
\hline Lines $\times$ Testers & 14 & 6.32 & 7.02 & $107^{*}$ & 46.4 & 117 & $73.6^{* *}$ & $3.18^{* *}$ \\
\hline Error & 34 & 6.78 & 8.06 & 87.9 & 56.2 & 30.5 & 44.3 & 3.25 \\
\hline \multicolumn{9}{|c|}{ Estimation of component of variance } \\
\hline$\sigma^{2} g$ (Line) & - & 0.103 & 0.583 & 4.08 & 2.46 & 2.463 & 26.1 & 0.016 \\
\hline$\sigma^{2} \mathrm{~g}$ (Tester) & - & 1.254 & 3.625 & 81.7 & 204 & 102 & 1.48 & -0.157 \\
\hline$\sigma^{2} \mathrm{gca}$ & - & 0.067 & 0.211 & 4.18 & 9.96 & 5.06 & 1.72 & -0.007 \\
\hline$\sigma^{2} \mathrm{sca}$ & - & -0.229 & -0.520 & 9.58 & -4.85 & 43.43 & 14.6 & -0.037 \\
\hline$\sigma^{2}$ gca $/ \sigma^{2} \mathrm{sca}$ & - & -0.292 & -0.405 & 0.43 & -2.05 & 0.117 & 0.118 & 0.176 \\
\hline
\end{tabular}

$\mathrm{DT}=$ Days to $50 \%$ tasseling, $\mathrm{DS}=$ Days to $50 \%$ silking, $\mathrm{PH}=$ plant height, $\mathrm{UCH}=\mathrm{Upper}$ cob height, $\mathrm{LCH}=\mathrm{Lower}$ cob height, WPCH $=$ Weight of per cob with husk, WHPCW= Weight of per cob without husk

Table 1. Cont'd.

\begin{tabular}{|c|c|c|c|c|c|c|c|c|}
\hline Source & df & CL (cm) & CD (cm) & NCPP & FYPP (g) & DFCH & IFLCH & CYPP (g) \\
\hline Replications & 1 & 1.04 & 0.024 & 0.103 & 403 & 88.4 & 84.9 & $61.62 * *$ \\
\hline Treatments & 34 & $1.26^{* *}$ & 0.132 & 0.362 & $125608 * *$ & 75.1 & $26.2 * *$ & $76.95 * *$ \\
\hline Parents & 10 & $2.23 *$ & 0.285 & 0.307 & 31708 & $33.0 *$ & 9.2 & $150.45 * *$ \\
\hline Parents vs Crosses & 1 & 1.92 & 0.397 & 0.248 & $3175113 * *$ & $1833^{*}$ & $345 * *$ & $116.49 * *$ \\
\hline Crosses & 23 & $0.814^{*}$ & 0.054 & 0.390 & $33846 *$ & $17.0 *$ & $19.8^{*}$ & $43.27 * *$ \\
\hline Lines & 7 & $0.723 * *$ & $0.059 *$ & $0.680 *$ & 19033 & $19.1 *$ & 31.0 & $45.83 * *$ \\
\hline Testers & 2 & 2.13 & $0.188^{*}$ & $0.007 *$ & $129752 * *$ & 47.7 & 25.4 & $8.67 * *$ \\
\hline Lines $\mathrm{x}$ Testers & 14 & 0.673 & $0.032 *$ & $0.300 *$ & $27552 * *$ & $11.6^{*}$ & 13.4 & $46.94 * *$ \\
\hline Error & 34 & 0.455 & 0.081 & 0.149 & 13890 & 7.6 & 18.7 & $57.13 * *$ \\
\hline
\end{tabular}




\begin{tabular}{|c|c|c|c|c|c|c|c|c|}
\hline Source & df & CL (cm) & CD (cm) & NCPP & FYPP (g) & DFCH & IFLCH & CYPP (g) \\
\hline \multicolumn{9}{|c|}{ Estimation of component of variance } \\
\hline$\sigma^{2} \mathrm{~g}($ Line $)$ & - & 0.008 & 0.004 & 0.063 & -1419.747 & 1.259 & 2.948 & -0.185 \\
\hline$\sigma^{2} \mathrm{~g}$ (Tester) & - & 0.091 & 0.010 & -0.018 & 6387.515 & 2.260 & 0.751 & -2.391 \\
\hline$\sigma^{2} \mathrm{gca}$ & - & 0.005 & 0.001 & 0.003 & 217.374 & 0.188 & 0.222 & -0.126 \\
\hline$\sigma^{2} \mathrm{sca}$ & - & 0.109 & -0.024 & 0.075 & 6831.070 & 2.001 & -2.662 & -5.093 \\
\hline$\sigma^{2} \mathrm{gca} / \sigma^{2} \mathrm{sca}$ & - & 0.045 & -0.031 & 0.041 & 0.032 & 0.094 & -0.083 & .0247 \\
\hline
\end{tabular}

$\mathrm{CL}=$ cob length, $\mathrm{CD}=$ cob diameter, $\mathrm{NCPP}=$ Number of cob per plant, $\mathrm{FYPP}=$ Fodder yield per plant, DFCH $=\mathrm{Days}$ to first cob harvest, $\mathrm{IFLCH}=\mathrm{Interval}$ between first and last cob harvest, $\mathrm{CYPP}=\mathrm{Cob}$ yield per Plant

\subsection{Proportional Contribution of Lines, Testers and Their Interactions}

The proportional contribution of lines was higher than testers and their interactions for weight of per cob with husk, number of cob per plant, interval between first and last cob harvest indicating their predominant maternal influence (Table 2). Testers exhibited less contribution to weight of per cob with husk, weight of per cob without husk, number of cob per plant, interval between first and last cob harvest and cob yield per plant. Motamedi et al., [8] found less influence of testers for kernel yield. The relative contribution of line $\times$ tester interaction was more important for days to $50 \%$ tasseling, cob length, con diameter, fodder yield per plant, days to first cob harvest and cob yield per plant. The higher contribution of interactions of the line $\times$ tester than lines and testers, indicating higher estimates of variances due to nonadditive genetic effects and the importance of specific combining ability.

Table 2. Proportional contribution (\%) of lines, testers and their interactions to total variance in baby corn.

\begin{tabular}{|c|c|c|c|c|c|c|c|}
\hline Source & DT & DS & PH (cm) & UCH (cm) & LCH (cm) & WPCH (g) & WPCH (g) \\
\hline Line & 25.6 & 24.4 & 17.5 & 5.6 & 15.2 & 56.8 & 33.3 \\
\hline Tester & 27.8 & 43.1 & 53.9 & 86.0 & 57.7 & 6.9 & 2.0 \\
\hline Line $\times$ Tester & 46.6 & 32.5 & 28.5 & 8.4 & 27.1 & 36.3 & 64.7 \\
\hline
\end{tabular}

Table 2. Cont'd.

\begin{tabular}{|c|c|c|c|c|c|c|c|}
\hline Source & CL (cm) & CD (cm) & NCPP & FYPP (g) & DFCP & IFLCH & CYPP (g) \\
\hline Line & 27.0 & 33.1 & 53.0 & 17.1 & 34.2 & 47.8 & 32.23 \\
\hline Tester & 22.7 & 30.3 & 0.1 & 33.3 & 24.4 & 11.2 & 1.74 \\
\hline Line $\times$ Tester & 50.3 & 36.6 & 46.8 & 49.5 & 41.4 & 41.1 & 66.03 \\
\hline
\end{tabular}

Abbreviations are given in Table 1

\subsection{General Combining Ability (GCA) Effects}

General combining ability is one of the main criteria of rapid genetic assaying of test genotypes under Line $\times$ Tester analysis. Selection of parents with good general combining ability is a prime requisite for any successful breeding program especially for heterosis breeding. The general combining ability effects and per se performance of parents (lines and testers) are presented in Table 3.

The GCA effects showed that line $\mathrm{KH}-101 / \mathrm{S}_{3}-32$ exhibited significant negative GCA effects for interval between $1^{\text {st }}$ and last cob harvest could be utilized for evolving earliness but it expressed highly significant negative GCA effects for number cob per plant. Only the line $\mathrm{KH}-101 / \mathrm{S}_{3}-1$ expressed highly significant positive GCA effects for number of cob per plant. $\mathrm{KH}-101 / \mathrm{S}_{3}-44$ expressed highly significant positive GCA effects for weight of per cob with husk. Dhasarathan et al. [11] and Rodrigues and Da Silva [16] also observed significant positive number of baby corns per plant. None of the parents showed significant positive GCA effects for cob diameter, which is supported by Rodrigues and Da Silva [16] and opposed by Dhasarathan et al. [11].

None of the parent showed significant positive GCA effects for cob yield per plant which is differing from the result of Dhasarathan et al. [11] and Rodrigues and Da Silva
[16]. It might due to using different genotypes. The tester $\mathrm{VS} / \mathrm{S}_{3}-24$ had positive significant effect on fodder yield per plant. The pollen parent $\mathrm{VS} / \mathrm{S}_{3}-2$ showed significant negative GCA for days to $50 \%$ tasseling, days to $50 \%$ silking and days to 1 st cob harvest, identified as early material. As GCA is generally associated with additive gene action in inheritance of characters, the lines and testers with high GCA may be utilized in hybridization program to improve a particular trait through transgressive segregation.

\subsection{Specific Combining Ability (SCA) Effects}

The Specific combining ability effects and mean of the crosses for cob yield and other qualitative characters are presented in Table 4. Positive SCA effect is expected for yield components. The crosses $\mathrm{KH}-101 / \mathrm{S}_{3}-44 \times \mathrm{VS} / \mathrm{S}_{3}-24$, $\mathrm{KH}-101 / \mathrm{S}_{3}-44 \times \mathrm{VS} / \mathrm{S}_{3}-25$,

$\mathrm{KH}-101 / \mathrm{S}_{3}-32 \times \mathrm{VS} / \mathrm{S}_{3}-24$ expressed highly significant positive SCA effects for number of cob per plant. The cross $\mathrm{KH}-101 / \mathrm{S}_{3}-3 \times \mathrm{VS} / \mathrm{S}_{3}-2$ recorded significant positive SCA for fodder yield per plant. The cross $\mathrm{KH}-101 / \mathrm{S} 3-1 \times \mathrm{VS} / \mathrm{S} 3-2$ exhibited significant negative plant height. These results were in harmony with the findings of Dhasarathan et al. [11] and Ahmed et al. [9] who reported significant positive SCA effects for cob yield per plant and significant negative SCA effects for plant height. 
Table 3. General combining ability effect of parents and their mean performance.

\begin{tabular}{|c|c|c|c|c|c|c|c|c|}
\hline \multirow{2}{*}{$\begin{array}{l}\text { Parents } \\
\text { Testers } \\
\end{array}$} & \multicolumn{2}{|l|}{ DT } & \multicolumn{2}{|l|}{ DS } & \multicolumn{2}{|l|}{ PH (cm) } & \multicolumn{2}{|c|}{ UCH (cm) } \\
\hline & GCA & Mean & GCA & Mean & GCA & Mean & GCA & Mean \\
\hline $\mathrm{VS} / \mathrm{S}_{3}-2$ & $-1.48 *$ & 92.5 & $-2.15 * *$ & 97 & $10.27 * *$ & 139.0 & -0.22 & 4.25 \\
\hline $\mathrm{VS} / \mathrm{S}_{3}-24$ & 0.83 & 94 & $1.85^{*}$ & 98 & -2.08 & 111.7 & 0.18 & 7.49 \\
\hline $\mathrm{VS} / \mathrm{S}_{3}-25$ & 0.65 & 91 & 0.29 & 96 & $-8.18^{* *}$ & 116.5 & 0.05 & 5.72 \\
\hline SE (gi) & 0.65 & & 0.71 & & 2.34 & & 0.45 & \\
\hline SE (gi-gj) & 0.92 & & 1.00 & & 3.32 & & 0.64 & \\
\hline \multicolumn{9}{|l|}{ Lines } \\
\hline $\mathrm{KH}-101 / \mathrm{S}_{3}-1$ & -0.04 & 90.5 & -1.06 & 95.0 & 1.06 & 97.3 & -1.02 & 32.6 \\
\hline $\mathrm{KH}-101 / \mathrm{S}_{3}-3$ & -1.71 & 83.0 & -1.73 & 90.0 & 5.10 & 107.0 & 2.01 & 37.5 \\
\hline $\mathrm{KH}-101 / \mathrm{S}_{3}-14$ & -0.38 & 94.5 & 0.10 & 98.5 & 0.66 & 86.8 & 1.41 & 40.3 \\
\hline $\mathrm{KH}-101 / \mathrm{S}_{3}-28$ & -0.04 & 90.0 & 0.44 & 97.0 & -0.44 & 127.0 & -0.03 & 60.7 \\
\hline KH-101/S ${ }_{3}-32$ & 1.63 & 90.0 & 0.94 & 97.0 & -5.10 & 91.2 & -3.76 & 37.3 \\
\hline $\mathrm{KH}-101 / \mathrm{S}_{3}-33$ & -1.04 & 87.5 & -1.56 & 94.5 & -8.14 & 102.0 & -5.26 & 42.5 \\
\hline $\mathrm{KH}-101 / \mathrm{S}_{3}-39$ & 0.63 & 87.0 & 0.94 & 93.5 & 5.66 & 120.0 & 3.81 & 54.4 \\
\hline $\mathrm{KH}-101 / \mathrm{S}_{3}-44$ & 0.96 & 87.0 & 1.94 & 93.5 & 1.20 & 121.0 & 2.84 & 52.5 \\
\hline SE & 1.06 & & 1.16 & & 3.83 & & 3.06 & \\
\hline SE (gi-gj) & 1.50 & & 1.64 & & 5.41 & & 4.33 & \\
\hline
\end{tabular}

Abbreviations are given in Table 1

Table 3. Cont'd.

\begin{tabular}{|c|c|c|c|c|c|c|}
\hline \multirow{2}{*}{$\begin{array}{l}\text { Parents } \\
\text { Testers }\end{array}$} & \multicolumn{2}{|c|}{ LEH. (cm) } & \multicolumn{2}{|c|}{ WPCH (g) } & \multicolumn{2}{|c|}{ WPCWH (g) } \\
\hline & GCA & Mean & GCA & Mean & GCA & Mean \\
\hline $\mathrm{VS} / \mathrm{S}_{3}-2$ & $10.88^{* *}$ & 34.4 & -2.41 & 31.70 & -0.22 & 4.25 \\
\hline $\mathrm{VS} / \mathrm{S}_{3}-24$ & -0.89 & 25.7 & 2.52 & 62.65 & 0.18 & 7.49 \\
\hline $\mathrm{VS} / \mathrm{S}_{3}-25$ & $-9.99 * *$ & 18.6 & -0.12 & 48.39 & 0.05 & 5.72 \\
\hline SE (gi) & 1.38 & & 1.66 & & 0.45 & \\
\hline SE (gi-gj) & 1.95 & & 2.35 & & 0.64 & \\
\hline \multicolumn{7}{|l|}{ Lines } \\
\hline $\mathrm{KH}-101 / \mathrm{S}_{3}-1$ & $-9.03 * *$ & 13.2 & -2.55 & 26.2 & -0.25 & 5.5 \\
\hline $\mathrm{KH}-101 / \mathrm{S}_{3}-3$ & 1.10 & 13.7 & $-6.29 *$ & 45.9 & -1.18 & 9.2 \\
\hline $\mathrm{KH}-101 / \mathrm{S}_{3}-14$ & 2.00 & 17.7 & 1.46 & 34.6 & -0.30 & 5.6 \\
\hline $\mathrm{KH}-101 / \mathrm{S}_{3}-28$ & 1.80 & 31.7 & -2.64 & 55.0 & 0.01 & 9.7 \\
\hline $\mathrm{KH}-101 / \mathrm{S}_{3}-32$ & 3.60 & 17.8 & -2.15 & 40.9 & 0.36 & 6.5 \\
\hline $\mathrm{KH}-101 / \mathrm{S}_{3}-33$ & $-5.60 *$ & 18.1 & -3.83 & 33.4 & -0.34 & 7.4 \\
\hline $\mathrm{KH}-101 / \mathrm{S}_{3}-39$ & 2.27 & 24.8 & 2.23 & 71.1 & 1.39 & 10.7 \\
\hline $\mathrm{KH}-101 / \mathrm{S}_{3}-44$ & 3.84 & 25.0 & $13.77 * *$ & 63.8 & 0.31 & 15.6 \\
\hline SE & 2.26 & & 2.72 & & 0.74 & \\
\hline SE (gi-gj) & 3.19 & & 3.84 & & 1.04 & \\
\hline
\end{tabular}

Abbreviations are given in Table 1

Table 3. Cont'd.

\begin{tabular}{|c|c|c|c|c|c|c|c|c|}
\hline \multirow{2}{*}{$\begin{array}{l}\text { Parents } \\
\text { Testers } \\
\end{array}$} & \multicolumn{2}{|c|}{ CL (cm) } & \multicolumn{2}{|c|}{ CD (cm) } & \multicolumn{2}{|c|}{ NCPP } & \multicolumn{2}{|c|}{ FYPP. (g) } \\
\hline & GCA & Mean & GCA & Mean & GCA & Mean & GCA & Mean \\
\hline $\mathrm{VS} / \mathrm{S}_{3}-2$ & $-0.37 *$ & 5.10 & -0.12 & 0.49 & 0.00 & 3.6 & 15.1 & 594.45 \\
\hline $\mathrm{VS} / \mathrm{S}_{3}-24$ & $0.36^{*}$ & 6.81 & 0.09 & 1.56 & 0.02 & 3.5 & $81.5^{* *}$ & 463.97 \\
\hline $\mathrm{VS} / \mathrm{S}_{3}-25$ & 0.01 & 6.63 & 0.03 & 0.85 & -0.02 & 3.6 & $-96.6 * *$ & 354.47 \\
\hline SE (gi) & 0.17 & & 0.07 & & 0.10 & & 29.4 & \\
\hline SE (gi-gj) & 0.24 & & 0.10 & & 0.14 & & 41.6 & \\
\hline \multicolumn{9}{|l|}{ Lines } \\
\hline $\mathrm{KH}-101 / \mathrm{S}_{3}-1$ & -0.48 & 4.8 & 0.13 & 0.6 & $0.54 * *$ & 2.6 & $-98.1^{*}$ & 134 \\
\hline $\mathrm{KH}-101 / \mathrm{S}_{3}-3$ & 0.14 & 7.1 & 0.00 & 1.2 & -0.07 & 3.2 & -14.6 & 215 \\
\hline $\mathrm{KH}-101 / \mathrm{S}_{3}-14$ & -0.02 & 6.3 & -0.15 & 0.7 & 0.00 & 3.0 & 24.2 & 337 \\
\hline $\mathrm{KH}-101 / \mathrm{S}_{3}-28$ & -0.25 & 7.6 & -0.03 & 1.2 & -0.13 & 3.3 & 33.2 & 389 \\
\hline $\mathrm{KH}-101 / \mathrm{S}_{3}-32$ & 0.09 & 6.3 & -0.01 & 1.4 & $-0.63 * *$ & 2.7 & 66.0 & 234 \\
\hline $\mathrm{KH}-101 / \mathrm{S}_{3}-33$ & -0.23 & 7.0 & -0.09 & 1.6 & 0.07 & 2.8 & -60.7 & 272 \\
\hline $\mathrm{KH}-101 / \mathrm{S}_{3}-39$ & $0.68 *$ & 8.1 & 0.01 & 1.3 & 0.27 & 3.3 & -1.4 & 319 \\
\hline $\mathrm{KH}-101 / \mathrm{S}_{3}-44$ & 0.07 & 7.9 & 0.14 & 1.3 & -0.03 & 2.6 & 51.3 & 382 \\
\hline SE (gi) & 0.28 & & 0.12 & & 0.16 & & 48.1 & \\
\hline SE (gi-gj) & 0.39 & & 0.16 & & 0.22 & & 68.0 & \\
\hline
\end{tabular}

Abbreviations are given in table 1 
Table 3. Cont'd.

\begin{tabular}{|c|c|c|c|c|c|c|}
\hline \multirow{2}{*}{$\begin{array}{l}\text { Parents } \\
\text { Testers } \\
\end{array}$} & \multicolumn{2}{|c|}{ DFCH } & \multicolumn{2}{|c|}{ IFLCH } & \multicolumn{2}{|c|}{ CYPP (g) } \\
\hline & GCA & Mean & GCA & Mean & GCA & Mean \\
\hline $\mathrm{VS} / \mathrm{S}_{3}-2$ & $-1.97 * *$ & 94.8 & 1.33 & 12.2 & -0.83 & 20.8 \\
\hline $\mathrm{VS} / \mathrm{S}_{3}-24$ & 1.27 & 98.9 & -1.18 & 11.1 & 0.56 & 21.1 \\
\hline $\mathrm{VS} / \mathrm{S}_{3}-25$ & 0.70 & 93.8 & -0.15 & 11.0 & 0.26 & 23.5 \\
\hline SE (gi) & 0.69 & & 1.08 & & 1.88 & \\
\hline SE (gi-gj) & 0.97 & & 1.53 & & 2.67 & \\
\hline \multicolumn{7}{|l|}{ Lines } \\
\hline $\mathrm{KH}-101 / \mathrm{S}_{3}-1$ & -0.19 & 99.1 & 0.29 & 9.4 & 2.14 & 24.8 \\
\hline $\mathrm{KH}-101 / \mathrm{S}_{3}-3$ & -1.25 & 88.1 & 1.04 & 15.7 & -3.20 & 22.5 \\
\hline $\mathrm{KH}-101 / \mathrm{S}_{3}-14$ & -0.65 & 102 & 0.67 & 7.5 & -0.99 & 23.2 \\
\hline $\mathrm{KH}-101 / \mathrm{S}_{3}-28$ & -0.62 & 98.3 & 1.67 & 9.2 & -0.69 & 20.9 \\
\hline $\mathrm{KH}-101 / \mathrm{S}_{3}-32$ & $2.65^{*}$ & 99.3 & $-5.19 * *$ & 10.2 & -2.66 & 29.4 \\
\hline $\mathrm{KH}-101 / \mathrm{S}_{3}-33$ & $-2.89 *$ & 92.3 & 2.10 & 12.7 & -0.26 & 14.3 \\
\hline $\mathrm{KH}-101 / \mathrm{S}_{3}-39$ & 1.18 & 92.5 & -0.66 & 10.2 & 5.48 & 25.2 \\
\hline $\mathrm{KH}-101 / \mathrm{S}_{3}-44$ & 1.78 & 95.5 & 0.08 & 10.2 & 0.18 & 48.6 \\
\hline SE (gi) & 1.12 & & 1.76 & & 3.08 & \\
\hline SE (gi-gj) & 1.59 & & 2.50 & & 4.36 & \\
\hline
\end{tabular}

Abbreviations are given in Table 1

Table 4. Specific combining ability effect and their mean performance.

\begin{tabular}{|c|c|c|c|c|c|c|c|c|}
\hline \multirow{2}{*}{ Crosses } & \multicolumn{2}{|c|}{ DT } & \multicolumn{2}{|c|}{ DS } & \multicolumn{2}{|c|}{ PH } & \multicolumn{2}{|c|}{ UCH.(cm) } \\
\hline & SCA & Mean & SCA & Mean & SCA & Mean & SCA & Mean \\
\hline $\mathrm{KH}-101 / \mathrm{S}_{3}-1 \times \mathrm{VS} / \mathrm{S}_{3}-2$ & -1.52 & 79.0 & -1.19 & 82.5 & $-13.88^{*}$ & 163 & -0.39 & 91 \\
\hline $\mathrm{KH}-101 / \mathrm{S}_{3}-1 \times \mathrm{VS} / \mathrm{S}_{3}-24$ & -1.33 & 81.5 & -1.19 & 86.5 & 9.09 & 174 & 0.00 & 88 \\
\hline $\mathrm{KH}-101 / \mathrm{S}_{3}-1 \times \mathrm{VS} / \mathrm{S}_{3}-25$ & 2.85 & 85.5 & 2.38 & 88.5 & 4.79 & 164 & 0.39 & 80 \\
\hline $\mathrm{KH}-101 / \mathrm{S}_{3}-3 \times \mathrm{VS} / \mathrm{S}_{3}-2$ & -0.85 & 78.0 & -0.52 & 82.5 & -0.11 & 181 & 1.46 & 106 \\
\hline $\mathrm{KH}-101 / \mathrm{S}_{3}-3 \times \mathrm{VS} / \mathrm{S}_{3}-24$ & -0.67 & 80.5 & -0.52 & 86.5 & 2.35 & 171 & -1.75 & 89 \\
\hline $\mathrm{KH}-101 / \mathrm{S}_{3}-3 \times \mathrm{VS} / \mathrm{S}_{3}-25$ & 1.52 & 82.5 & 1.04 & 86.5 & -2.25 & 161 & 0.30 & 73 \\
\hline $\mathrm{KH}-101 / \mathrm{S}_{3}-14 \times \mathrm{VS} / \mathrm{S}_{3}-2$ & -0.69 & 79.5 & -0.85 & 84.0 & -2.28 & 175 & -0.35 & 105 \\
\hline $\mathrm{KH}-101 / \mathrm{S}_{3}-14 \times \mathrm{VS} / \mathrm{S}_{3}-24$ & -0.50 & 82.0 & -0.85 & 88.0 & 0.59 & 165 & 0.48 & 81 \\
\hline $\mathrm{KH}-101 / \mathrm{S}_{3}-14 \times \mathrm{VS} / \mathrm{S}_{3}-25$ & 1.19 & 83.5 & 1.71 & 89.0 & 1.69 & 160 & -0.13 & 80 \\
\hline $\mathrm{KH}-101 / \mathrm{S}_{3}-28 \times \mathrm{VS} / \mathrm{S}_{3}-2$ & -0.02 & 80.5 & -0.19 & 85.0 & 5.52 & 181 & -0.76 & 104 \\
\hline $\mathrm{KH}-101 / \mathrm{S}_{3}-28 \times \mathrm{VS} / \mathrm{S}_{3}-24$ & -0.33 & 82.5 & -1.19 & 88.0 & -3.31 & 160 & 0.90 & 84 \\
\hline $\mathrm{KH}-101 / \mathrm{S}_{3}-28 \times \mathrm{VS} / \mathrm{S}_{3}-25$ & 0.35 & 83.0 & 1.38 & 89.0 & -2.21 & 155 & -0.14 & 74 \\
\hline $\mathrm{KH}-101 / \mathrm{S}_{3}-32 \times \mathrm{VS} / \mathrm{S}_{3}-2$ & 1.81 & 84.0 & 1.31 & 87.0 & 9.49 & 181 & -0.43 & 104 \\
\hline $\mathrm{KH}-101 / \mathrm{S}_{3}-32 \times \mathrm{VS} / \mathrm{S}_{3}-24$ & 1.00 & 85.5 & 1.31 & 91.0 & -4.15 & 155 & 1.28 & 80 \\
\hline $\mathrm{KH}-101 / \mathrm{S}_{3}-32 \times \mathrm{VS} / \mathrm{S}_{3}-25$ & -2.81 & 81.5 & -2.63 & 85.5 & -5.35 & 147 & -0.85 & 67 \\
\hline $\mathrm{KH}-101 / \mathrm{S}_{3}-33 \times \mathrm{VS} / \mathrm{S}_{3}-2$ & -1.02 & 78.5 & -1.69 & 81.5 & -7.08 & 161 & -0.24 & 96 \\
\hline $\mathrm{KH}-101 / \mathrm{S}_{3}-33 \times \mathrm{VS} / \mathrm{S}_{3}-24$ & 1.17 & 83.0 & 1.31 & 88.5 & 2.89 & 159 & 0.39 & 79 \\
\hline $\mathrm{KH}-101 / \mathrm{S}_{3}-33 \times \mathrm{VS} / \mathrm{S}_{3}-25$ & -0.15 & 81.5 & 0.38 & 86.0 & 4.19 & 154 & -0.16 & 71 \\
\hline $\mathrm{KH}-101 / \mathrm{S}_{3}-39 \times \mathrm{VS} / \mathrm{S}_{3}-2$ & 2.31 & 83.5 & 2.31 & 88.0 & 10.23 & 192 & 0.37 & 112 \\
\hline $\mathrm{KH}-101 / \mathrm{S}_{3}-39 \times \mathrm{VS} / \mathrm{S}_{3}-24$ & -0.50 & 83.0 & 0.31 & 90.0 & -6.21 & 163 & 0.99 & 87 \\
\hline $\mathrm{KH}-101 / \mathrm{S}_{3}-39 \times \mathrm{VS} / \mathrm{S}_{3}-25$ & -1.81 & 81.5 & -2.63 & 85.5 & -4.01 & 159 & -1.36 & 74 \\
\hline $\mathrm{KH}-101 / \mathrm{S}_{3}-44 \times \mathrm{VS} / \mathrm{S}_{3}-2$ & -0.02 & 81.5 & 0.81 & 87.5 & -1.91 & 175 & 0.34 & 107 \\
\hline $\mathrm{KH}-101 / \mathrm{S}_{3}-44 \times \mathrm{VS} / \mathrm{S}_{3}-24$ & 1.17 & 85.0 & 0.81 & 91.5 & -1.25 & 164 & -2.28 & 86 \\
\hline $\mathrm{KH}-101 / \mathrm{S}_{3}-44 \times \mathrm{VS} / \mathrm{S}_{3}-25$ & -1.15 & 82.5 & -1.63 & 87.5 & 3.15 & 162 & 1.95 & 78 \\
\hline $\mathrm{SE}_{(s i j)}$ & 1.84 & & 2.01 & & 6.63 & & 1.28 & \\
\hline S.E. $(s i j-s k l)$ & 2.60 & & 2.84 & & 9.38 & & 1.80 & \\
\hline
\end{tabular}

Abbreviations are given in Table 1

Table 4. Cont'd.

\begin{tabular}{|c|c|c|c|c|c|c|}
\hline \multirow{2}{*}{ Crosses } & \multicolumn{2}{|c|}{ LCH (cm) } & \multicolumn{2}{|c|}{ WCPH (g) } & \multicolumn{2}{|c|}{ WPCWH (g) } \\
\hline & SCA & Mean & SCA & Mean & SCA & Mean \\
\hline $\mathrm{KH}-101 / \mathrm{S}_{3}-1 \times \mathrm{VS} / \mathrm{S}_{3}-2$ & $-17.5 * *$ & 34.3 & 0.35 & 45.0 & -1.52 & 79.0 \\
\hline $\mathrm{KH}-101 / \mathrm{S}_{3}-1 \times \mathrm{VS} / \mathrm{S}_{3}-24$ & 7.49 & 47.6 & 1.80 & 51.4 & -1.33 & 81.5 \\
\hline $\mathrm{KH}-101 / \mathrm{S}_{3}-1 \times \mathrm{VS} / \mathrm{S}_{3}-25$ & $10.09 *$ & 41.1 & -2.15 & 44.8 & 2.85 & 85.5 \\
\hline $\mathrm{KH}-101 / \mathrm{S}_{3}-3 \times \mathrm{VS} / \mathrm{S}_{3}-2$ & 1.88 & 63.9 & $10.83^{*}$ & 51.8 & -0.85 & 78.0 \\
\hline $\mathrm{KH}-101 / \mathrm{S}_{3}-3 \times \mathrm{VS} / \mathrm{S}_{3}-24$ & 2.16 & 52.4 & $-13.46^{*}$ & 32.4 & -0.67 & 80.5 \\
\hline $\mathrm{KH}-101 / \mathrm{S}_{3}-3 \times \mathrm{VS} / \mathrm{S}_{3}-25$ & -4.04 & 37.1 & 2.62 & 45.9 & 1.52 & 82.5 \\
\hline $\mathrm{KH}-101 / \mathrm{S}_{3}-14 \times \mathrm{VS} / \mathrm{S}_{3}-2$ & -2.92 & 60.0 & -3.47 & 45.2 & -0.69 & 79.5 \\
\hline $\mathrm{KH}-101 / \mathrm{S}_{3}-14 \times \mathrm{VS} / \mathrm{S}_{3}-24$ & -0.14 & 51.0 & 5.19 & 58.8 & -0.50 & 82.0 \\
\hline $\mathrm{KH}-101 / \mathrm{S}_{3}-14 \times \mathrm{VS} / \mathrm{S}_{3}-25$ & 3.06 & 45.1 & -1.72 & 49.3 & 1.19 & 83.5 \\
\hline $\mathrm{KH}-101 / \mathrm{S}_{3}-28 \times \mathrm{VS} / \mathrm{S}_{3}-2$ & 0.08 & 62.8 & -1.77 & 42.8 & -0.02 & 80.5 \\
\hline
\end{tabular}




\begin{tabular}{|c|c|c|c|c|c|c|}
\hline \multirow{2}{*}{ Crosses } & \multicolumn{2}{|c|}{ LCH (cm) } & \multicolumn{2}{|c|}{ WCPH (g) } & \multicolumn{2}{|c|}{$\overline{\text { WPCWH (g) }}$} \\
\hline & SCA & Mean & SCA & Mean & SCA & Mean \\
\hline $\mathrm{KH}-101 / \mathrm{S}_{3}-28 \times \mathrm{VS} / \mathrm{S}_{3}-24$ & -4.64 & 46.3 & -1.20 & 48.3 & -0.33 & 82.5 \\
\hline $\mathrm{KH}-101 / \mathrm{S}_{3}-28 \times \mathrm{VS} / \mathrm{S}_{3}-25$ & 4.56 & 46.4 & 2.97 & 49.9 & 0.35 & 83.0 \\
\hline $\mathrm{KH}-101 / \mathrm{S}_{3}-32 \times \mathrm{VS} / \mathrm{S}_{3}-2$ & 7.28 & 71.8 & -5.85 & 39.2 & 1.81 & 84.0 \\
\hline $\mathrm{KH}-101 / \mathrm{S}_{3}-32 \times \mathrm{VS} / \mathrm{S}_{3}-24$ & -3.14 & 49.6 & 7.43 & 57.4 & 1.00 & 85.5 \\
\hline $\mathrm{KH}-101 / \mathrm{S}_{3}-32 \times \mathrm{VS} / \mathrm{S}_{3}-25$ & -4.14 & 39.5 & -1.57 & 45.8 & -2.81 & 81.5 \\
\hline $\mathrm{KH}-101 / \mathrm{S}_{3}-33 \times \mathrm{VS} / \mathrm{S}_{3}-2$ & -1.72 & 53.6 & 1.67 & 45.1 & -1.02 & 78.5 \\
\hline $\mathrm{KH}-101 / \mathrm{S}_{3}-33 \times \mathrm{VS} / \mathrm{S}_{3}-24$ & 1.36 & 44.9 & 1.21 & 49.5 & 1.17 & 83.0 \\
\hline $\mathrm{KH}-101 / \mathrm{S}_{3}-33 \times \mathrm{VS} / \mathrm{S}_{3}-25$ & 0.36 & 34.8 & -2.88 & 42.8 & -0.15 & 81.5 \\
\hline $\mathrm{KH}-101 / \mathrm{S}_{3}-39 \times \mathrm{VS} / \mathrm{S}_{3}-2$ & $8.42 *$ & 71.6 & -3.51 & 46.0 & 2.31 & 83.5 \\
\hline $\mathrm{KH}-101 / \mathrm{S}_{3}-39 \times \mathrm{VS} / \mathrm{S}_{3}-24$ & 0.49 & 51.9 & -1.15 & 53.2 & -0.50 & 83 \\
\hline $\mathrm{KH}-101 / \mathrm{S}_{3}-39 \times \mathrm{VS} / \mathrm{S}_{3}-25$ & $-8.91 *$ & 33.4 & 4.65 & 56.4 & -1.81 & 81.5 \\
\hline $\mathrm{KH}-101 / \mathrm{S}_{3}-44 \times \mathrm{VS} / \mathrm{S}_{3}-2$ & 4.55 & 69.3 & 1.75 & 62.8 & -0.02 & 81.5 \\
\hline $\mathrm{KH}-101 / \mathrm{S}_{3}-44 \times \mathrm{VS} / \mathrm{S}_{3}-24$ & -3.57 & 49.4 & 0.17 & 66.1 & 1.17 & 85 \\
\hline $\mathrm{KH}-101 / \mathrm{S}_{3}-44 \times \mathrm{VS} / \mathrm{S}_{3}-25$ & -0.97 & 42.9 & -1.92 & 61.4 & -1.15 & 82.5 \\
\hline $\mathrm{SE}_{(s i i)}$ & 3.91 & & 4.71 & & 1.84 & \\
\hline S.E. $(s i j-s k l)$ & 5.52 & & 6.66 & & 2.60 & \\
\hline
\end{tabular}

Abbreviations are given in table 1

Table 4. Cont'd

\begin{tabular}{|c|c|c|c|c|c|c|c|c|}
\hline \multirow{2}{*}{ Crosses } & \multicolumn{2}{|c|}{ CL (cm) } & \multicolumn{2}{|c|}{ CD (cm) } & \multicolumn{2}{|c|}{ NCPP } & \multicolumn{2}{|c|}{ FYPP. (g) } \\
\hline & SCA & Mean & SCA & Mean & SCA & Mean & SCA & Mean \\
\hline $\mathrm{KH}-101 / \mathrm{S}_{3}-1 \times \mathrm{VS} / \mathrm{S}_{3}-2$ & 0.21 & 6.42 & -0.08 & 0.88 & 0.72 & 4.5 & -161 & 551 \\
\hline $\mathrm{KH}-101 / \mathrm{S}_{3}-1 \times \mathrm{VS} / \mathrm{S}_{3}-24$ & -0.31 & 6.63 & 0.05 & 1.21 & -0.27 & 3.5 & 114 & 893 \\
\hline $\mathrm{KH}-101 / \mathrm{S}_{3}-1 \times \mathrm{VS} / \mathrm{S}_{3}-25$ & 0.09 & 6.67 & 0.03 & 1.13 & -0.45 & 3.3 & 46.4 & 646 \\
\hline $\mathrm{KH}-101 / \mathrm{S}_{3}-3 \times \mathrm{VS} / \mathrm{S} 3-2$ & 0.46 & 7.28 & 0.02 & 0.85 & 0.33 & 3.5 & $193^{*}$ & 988 \\
\hline $\mathrm{KH}-101 / \mathrm{S}_{3}-3 \times \mathrm{VS} / \mathrm{S}_{3}-24$ & -0.18 & 7.37 & -0.13 & 0.91 & $-0.19 * *$ & 3.0 & -81.4 & 780 \\
\hline $\mathrm{KH}-101 / \mathrm{S}_{3}-3 \times \mathrm{VS} / \mathrm{S}_{3}-25$ & -0.28 & 6.92 & 0.10 & 1.08 & $-0.14 * *$ & 3.0 & -111 & 572 \\
\hline $\mathrm{KH}-101 / \mathrm{S}_{3}-14 \times \mathrm{VS} / \mathrm{S}_{3}-2$ & 0.24 & 6.90 & 0.04 & 0.71 & 0.16 & 3.4 & $-199 *$ & 635 \\
\hline $\mathrm{KH}-101 / \mathrm{S}_{3}-14 \times \mathrm{VS} / \mathrm{S}_{3}-24$ & 0.12 & 7.52 & -0.14 & 0.75 & -0.05 & 3.2 & 34.3 & 935 \\
\hline $\mathrm{KH}-101 / \mathrm{S}_{3}-14 \times \mathrm{VS} / \mathrm{S}_{3}-25$ & -0.36 & 6.68 & 0.10 & 0.93 & $-0.11 * *$ & 3.1 & 164 & 887 \\
\hline $\mathrm{KH}-101 / \mathrm{S}_{3}-28 \times \mathrm{VS} / \mathrm{S}_{3}-2$ & -0.16 & 6.28 & -0.03 & 0.76 & 0.00 & 3.1 & 22.3 & 865 \\
\hline $\mathrm{KH}-101 / \mathrm{S}_{3}-28 \times \mathrm{VS} / \mathrm{S}_{3}-24$ & 0.09 & 7.25 & -0.04 & 0.96 & 0.28 & 3.4 & -17.3 & 892 \\
\hline $\mathrm{KH}-101 / \mathrm{S}_{3}-28 \times \mathrm{VS} / \mathrm{S}_{3}-25$ & 0.06 & 6.88 & 0.07 & 1.01 & -0.28 & 2.8 & -5.0 & 726 \\
\hline $\mathrm{KH}-101 / \mathrm{S}_{3}-32 \times \mathrm{VS} / \mathrm{S}_{3}-2$ & 0.22 & 6.99 & -0.06 & 0.75 & -0.30 & 2.3 & 54.8 & 931 \\
\hline $\mathrm{KH}-101 / \mathrm{S}_{3}-32 \times \mathrm{VS} / \mathrm{S}_{3}-24$ & 0.01 & 7.51 & 0.11 & 1.13 & $0.08 * *$ & 2.7 & -5.5 & 937 \\
\hline $\mathrm{KH}-101 / \mathrm{S}_{3}-32 \times \mathrm{VS} / \mathrm{S}_{3}-25$ & -0.23 & 6.92 & -0.05 & 0.91 & 0.22 & 2.8 & -49.3 & 715 \\
\hline $\mathrm{KH}-101 / \mathrm{S}_{3}-33 \times \mathrm{VS} / \mathrm{S}_{3}-2$ & 0.18 & 6.63 & 0.05 & 0.78 & $-0.10^{*}$ & 3.2 & -44.1 & 705 \\
\hline $\mathrm{KH}-101 / \mathrm{S}_{3}-33 \times \mathrm{VS} / \mathrm{S}_{3}-24$ & -0.39 & 6.79 & -0.06 & 0.88 & $-0.12 *$ & 3.2 & 31.6 & 847 \\
\hline $\mathrm{KH}-101 / \mathrm{S}_{3}-33 \times \mathrm{VS} / \mathrm{S}_{3}-25$ & 0.21 & 7.04 & 0.01 & 0.89 & 0.22 & 3.5 & 12.5 & 650 \\
\hline $\mathrm{KH}-101 / \mathrm{S}_{3}-39 \times \mathrm{VS} / \mathrm{S}_{3}-2$ & 0.35 & 7.72 & 0.15 & 0.99 & $-0.20 * *$ & 3.3 & 88.7 & 897 \\
\hline $\mathrm{KH}-101 / \mathrm{S}_{3}-39 \times \mathrm{VS} / \mathrm{S}_{3}-24$ & 0.01 & 8.10 & -0.05 & 1.00 & $-0.12 *$ & 3.4 & -22.7 & 852 \\
\hline $\mathrm{KH}-101 / \mathrm{S}_{3}-39 \times \mathrm{VS} / \mathrm{S}_{3}-25$ & -0.36 & 7.38 & -0.10 & 0.89 & 0.32 & 3.8 & -66.0 & 631 \\
\hline $\mathrm{KH}-101 / \mathrm{S}_{3}-44 \times \mathrm{VS} / \mathrm{S}_{3}-2$ & -1.50 & 5.25 & -0.09 & 0.88 & -0.60 & 2.6 & 45.4 & 907 \\
\hline $\mathrm{KH}-101 / \mathrm{S}_{3}-44 \times \mathrm{VS} / \mathrm{S}_{3}-24$ & 0.64 & 8.13 & 0.25 & 1.42 & $0.38 * *$ & 3.6 & 53.7 & 874 \\
\hline $\mathrm{KH}-101 / \mathrm{S}_{3}-44 \times \mathrm{VS} / \mathrm{S}_{3}-25$ & 0.86 & 7.99 & -0.16 & 0.95 & $0.22 * *$ & 3.4 & 8.3 & 758 \\
\hline $\mathrm{SE}_{(s i j)}$ & 0.48 & & 0.20 & & 0.27 & & 83.34 & \\
\hline S.E. $(s i i-s k l)$ & 0.67 & & 0.28 & & 0.39 & & 117.85 & \\
\hline
\end{tabular}

Abbreviations are given in Table 1

Table 4. Cont'd

\begin{tabular}{|c|c|c|c|c|c|c|}
\hline \multirow{2}{*}{ Crosses } & \multicolumn{2}{|c|}{ DFCH } & \multicolumn{2}{|c|}{ IFLCH } & \multicolumn{2}{|c|}{ CYPP (g) } \\
\hline & SCA & Mean & SCA & Mean & SCA & Mean \\
\hline $\mathrm{KH}-101 / \mathrm{S}_{3}-1 \times \mathrm{VS} / \mathrm{S}_{3}-2$ & -0.80 & 81.9 & 2.61 & 19.9 & 3.33 & 26.8 \\
\hline $\mathrm{KH}-101 / \mathrm{S}_{3}-1 \times \mathrm{VS} / \mathrm{S}_{3}-24$ & -2.84 & 83.1 & 1.16 & 15.9 & -1.87 & 23.0 \\
\hline $\mathrm{KH}-101 / \mathrm{S}_{3}-1 \times \mathrm{VS} / \mathrm{S}_{3}-25$ & 3.64 & 89.0 & -3.77 & 12.0 & -1.47 & 23.1 \\
\hline $\mathrm{KH}-101 / \mathrm{S}_{3}-3 \times \mathrm{VS} / \mathrm{S}_{3}-2$ & 1.57 & 83.2 & -0.89 & 17.1 & 8.08 & 26.2 \\
\hline $\mathrm{KH}-101 / \mathrm{S}_{3}-3 \times \mathrm{VS} / \mathrm{S}_{3}-24$ & -1.67 & 83.2 & 0.06 & 15.6 & -6.76 & 12.76 \\
\hline $\mathrm{KH}-101 / \mathrm{S}_{3}-3 \times \mathrm{VS} / \mathrm{S}_{3}-25$ & 0.10 & 84.4 & 0.83 & 17.4 & -1.32 & 17.9 \\
\hline $\mathrm{KH}-101 / \mathrm{S}_{3}-14 \times \mathrm{VS} / \mathrm{S}_{3}-2$ & -1.23 & 81.0 & -1.38 & 16.3 & -0.23 & 20.1 \\
\hline $\mathrm{KH}-101 / \mathrm{S}_{3}-14 \times \mathrm{VS} / \mathrm{S}_{3}-24$ & -0.17 & 85.3 & 1.83 & 17.0 & 1.87 & 23.6 \\
\hline $\mathrm{KH}-101 / \mathrm{S}_{3}-14 \times \mathrm{VS} / \mathrm{S}_{3}-25$ & 1.40 & 86.3 & -0.45 & 15.7 & -1.63 & 19.8 \\
\hline $\mathrm{KH}-101 / \mathrm{S}_{3}-28 \times \mathrm{VS} / \mathrm{S}_{3}-2$ & -0.07 & 82.2 & 1.68 & 20.3 & -2.53 & 18.1 \\
\hline $\mathrm{KH}-101 / \mathrm{S}_{3}-28 \times \mathrm{VS} / \mathrm{S}_{3}-24$ & -2.00 & 83.5 & -0.78 & 15.3 & 5.47 & 27.5 \\
\hline
\end{tabular}




\begin{tabular}{|c|c|c|c|c|c|c|}
\hline \multirow{2}{*}{ Crosses } & \multicolumn{2}{|c|}{ DFCH } & \multicolumn{2}{|c|}{ IFLCH } & \multicolumn{2}{|c|}{ CYPP (g) } \\
\hline & SCA & Mean & SCA & Mean & SCA & Mean \\
\hline $\mathrm{KH}-101 / \mathrm{S}_{3}-28 \times \mathrm{VS} / \mathrm{S}_{3}-25$ & 2.07 & 87.0 & -0.90 & 16.3 & -2.93 & 18.8 \\
\hline $\mathrm{KH}-101 / \mathrm{S}_{3}-32 \times \mathrm{VS} / \mathrm{S}_{3}-2$ & 0.67 & 86.2 & -3.16 & 8.60 & -3.67 & 15.0 \\
\hline $\mathrm{KH}-101 / \mathrm{S}_{3}-32 \times \mathrm{VS} / \mathrm{S}_{3}-24$ & 3.13 & 91.9 & 0.34 & 9.60 & 2.33 & 22.4 \\
\hline $\mathrm{KH}-101 / \mathrm{S}_{3}-32 \times \mathrm{VS} / \mathrm{S}_{3}-25$ & -3.80 & 84.4 & 2.82 & 13.1 & 1.33 & 21.1 \\
\hline $\mathrm{KH}-101 / \mathrm{S}_{3}-33 \times \mathrm{VS} / \mathrm{S}_{3}-2$ & -1.00 & 79.0 & -0.43 & 18.6 & -1.87 & 19.2 \\
\hline $\mathrm{KH}-101 / \mathrm{S}_{3}-33 \times \mathrm{VS} / \mathrm{S}_{3}-24$ & 1.26 & 84.5 & 2.95 & 19.5 & 0.03 & 22.5 \\
\hline $\mathrm{KH}-101 / \mathrm{S}_{3}-39 \times \mathrm{VS} / \mathrm{S}_{3}-2$ & 1.83 & 85.9 & 0.81 & 17.1 & -0.20 & 26.6 \\
\hline $\mathrm{KH}-101 / \mathrm{S}_{3}-39 \times \mathrm{VS} / \mathrm{S}_{3}-24$ & 0.90 & 88.2 & -3.49 & 10.3 & 3.80 & 32.0 \\
\hline $\mathrm{KH}-101 / \mathrm{S}_{3}-39 \times \mathrm{VS} / \mathrm{S}_{3}-25$ & -2.73 & 84.0 & 2.68 & 17.5 & -3.60 & 24.3 \\
\hline $\mathrm{KH}-101 / \mathrm{S}_{3}-44 \times \mathrm{VS} / \mathrm{S}_{3}-2$ & -0.97 & 83.7 & 0.77 & 17.8 & -2.91 & 18.6 \\
\hline $\mathrm{KH}-101 / \mathrm{S}_{3}-44 \times \mathrm{VS} / \mathrm{S}_{3}-24$ & 1.40 & 89.3 & -2.08 & 12.5 & 8.88 & 28.02 \\
\hline $\mathrm{KH}-101 / \mathrm{S}_{3}-44 \times \mathrm{VS} / \mathrm{S}_{3}-25$ & -0.43 & 86.9 & 1.31 & 16.9 & 7.79 & 30.4 \\
\hline $\mathrm{SE}_{(s i j)}$ & 1.95 & & 3.05 & & 5.34 & \\
\hline
\end{tabular}

Abbreviations are given in Table 1

\section{Conclusion}

Considering the above result the parent $\mathrm{KH}-101 / \mathrm{S}_{3}-1$, KH$101 / S_{3}-44$ identified as a good general combiner for number of cob per plant and weight of per cob with husk respectively. Furthermore, considering days to tasseling, silking, plant height, lower ear height, upper ear height, number of cob per plant and cob yield per plant, based on mean performance and SCA effects the two crosses namely $\mathrm{KH}-101 / \mathrm{S}_{3}$ $44 \times \mathrm{VS} / \mathrm{S}_{3}-24, \quad \mathrm{KH}-101 / \mathrm{S}_{3}-44 \times \mathrm{VS} / \mathrm{S}_{3}-25$ were selected as promising hybrid, could be used in future breeding program to develop high yielding baby corn hybrids with desirable qualities.

\section{References}

[1] Carol AM, Zenz L. 1998. Vegetable Research and Extension. Mount Vernon Northwestern Washington Research and Extension Center, Washington State University Extension. 360 NW North St., Chehalis, WA 98532.

[2] Chauhan SK, Mohan J. 2010. Estimates of variability, heritability and genetic advance in baby corn. Indian J. Hort 67: $238-241$.

[3] Dhasarathan M, Babu C, Iyanar K, Velayudham, K. 2012. Studies on genetic potential of baby corn (Zea mays L.) hybrids for yield and quality traits. Electronic J. Plant Breed 3: $853-860$.

[4] Kempthorne O. 1957. An Introduction to Genetic Statistics. New York: John Wiley \& Sons, Inc. London: Chapman \& Hall Ltd. pp. 458-471.

[5] Rodrigues F, Pinho RGV, Albuquerque CJB, Filho EMF, Goulart JC. 2009. Capacidade de combinação entre linhagens de milhovisando à produção de milhoverde. Bragantia 68: 7584.

[6] Ceyhan E, Avci M, Karada S. 2008. Line $\times$ tester analysis in pea (Pisum sativum L.): Identification of superior parents for seed yield and its components. African J. Biotech 7 (16): 2810-2817.
[7] Kanagarasu S, Nallathambi G, Ganesan KN. 2010. Combining ability analysis for yield and its component traits in maize (Zea mays L.). Electronic J. Plant Breeding 1 (4): 915-920.

[8] Motamedi M, Choukan R, Hervan E, Bihamta, MR, Kajouri FD. 2014. Investigation of genetic control for yield and related traits in maize (Zea mays L.) lines derived from temperate and sub-tropical germplasm. Int. J. Bio. Sciences 5 (12): 123-129.

[9] Ahmed A, Begum S, Omy SH, Rohman MM. Amiruzzaman M. 2015. Evaluation of inbred lines of baby com through line $\times$ tester method. Bangladesh J. Agril. Res 41 (2): 311-321.

[10] Suneetha Y, Patel JR, Srinivas T. 2000. Studies on combining ability for forage characters in maize (Zea mays L.). Crop Res 9: 226-270.

[11] Dhasarathan M, Babu C, Iyanar K. 2015. Combining ability and gene action studies for yield and quality traits in baby corn (Zea mays L.). SABRAO J. Breed. Gen 47 (1): 60-69.

[12] Anantha, MS. 2004. Combining ability and molecular diversity analysis in maize inbreds. M.Sc. (Ag.), Thesis, Tamil Nadu Agricultural University, India.

[13] Selvarani E. 2007. Studies on combining ability of fodder maize (Zea mays L.) and sweet corn (Zea mays L. Saccharata) for evolving dual purpose maize genotypes. M.Sc. (Ag.), Thesis, Tamil Nadu Agricultural University, Coimbatore.

[14] Geetha K, Jayaraman. 2000. Genetic analysis of yield in maize. Madras Agric. J 87 (10-12): 638-640.

[15] Prakash S, Ganguly DK. 2004. Combining ability for various yield component characters in maize (Zea mays L.). J. Res. (BAU) 16 (1): 55-60.

[16] Rodrigues LRF, Da Silva N. 2002. Combining ability in baby corn inbred lines (Zea mays L.). Crop Breed. Applied Biotech 2 (3): $361-368$

[17] Mahto RN, Ganguly DK. 2001. Heterosis and combining ability studies in maize (Zea mays L.). J. Res. Brirsa Agric. Univ 13: 197-199. 